\title{
Extending the Resynchronization Attack ${ }^{\star}$
}

\author{
Frederik Armknecht ${ }^{1, \star \star}$, Joseph Lano ${ }^{2, \star \star \star}$, and Bart Preneel ${ }^{2}$ \\ 1 Universität Mannheim, Theoretische Informatik, \\ 68131 Mannheim, Germany \\ armknecht@th.informatik.uni-mannheim.de \\ ${ }^{2}$ Katholieke Universiteit Leuven, \\ Dept. Elect. Eng.-ESAT/SCD-COSIC, \\ Kasteelpark Arenberg 10, 3001 Heverlee, Belgium \\ \{joseph.lano, bart.preneel\}@esat.kuleuven.ac.be
}

\begin{abstract}
Synchronous stream ciphers need perfect synchronization between sender and receiver. In practice, this is ensured by a resync mechanism. Daemen et al. 10 first described attacks on ciphers using such a resync mechanism. In this paper, we extend their attacks in several ways by combining the standard attack with cryptanalytic techniques such as algebraic attacks and linear cryptanalysis. Our results show that using linear resync mechanisms should be avoided, and provide lower bounds for the nonlinearity required from a secure resync mechanism.
\end{abstract}

\section{Introduction}

Synchronous stream ciphers generate a key stream independently from the plaintext. They typically consist of a finite state machine from which at each iteration a key stream bit is generated by an output function. Synchronous stream ciphers have the advantage that there is no error propagation. On the other hand, perfect synchronization between sender and receiver is required. In order to prevent synchronization loss or to restore synchronization after synchronization loss is detected, a resynchronization mechanism is used. Such a mechanism generates a new initial state for the finite state machine from the secret key and a unique initialization vector $I V$ and thus prevents the reuse of key stream. For the sake of efficiency the resynchronization mechanism should be as fast as possible.

Daemen, Govaerts and Vandewalle [10] observed that this resynchronization mechanism can lead to a new type of attacks on synchronous stream ciphers. They also showed an efficient attack on nonlinearly filtered systems using a linear resynchronization mechanism and using an output Boolean function with few

* This work was supported by the Concerted Research Action (GOA) Mefisto2000/04 of the Flemish Government.

** This work has been supported by grant 620307 of the DFG (German Research Foundation).

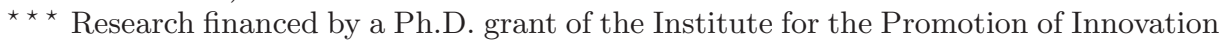
through Science and Technology in Flanders (IWT-Vlaanderen). 
inputs. Golic and Morgari [13] extended this attack to the case where the output function is unknown. Borissov et al. 7] showed that a ciphertext-only attack is also possible in some cases.

In this paper, we extend the resynchronization attack to overcome some limitations of the original attack of Daemen et al. We achieve this by further refining the original resynchronization attack and by combining the attack with other attack methodologies, notably with algebraic attacks. We do not show practical applications of our attacks in this article, but describe such attacks on E0, A5 and the summation generator in the extended version of our paper [2].

The paper is organized as follows. In Sect. 2, we present some preliminary notions: Boolean functions, the general framework of a stream cipher, algebraic attacks and resynchronization attacks. In Sect. 3 we present the Daemen et al. attack and its limitations. In Sect. 4 we show how to perform the Daemen et al. attack in real-time by precomputation. In Sect. 5 we describe several methods to mount a resync attack when the number of resyncs is small. Section [6 describes methods to mount attacks when the output function has many inputs. In Sect. 7, we describe attacks on stream ciphers with memory and in Sect. 8 we discuss attacks on stream ciphers with nonlinear resynchronization mechanism. We conclude in Sect. 9

\section{Preliminaries}

\subsection{Boolean Functions and Related Inputs}

In this section we repeat some definitions and known facts about Boolean functions. Additionally, we provide some theorems about Boolean functions and related inputs. All calculations are done over the finite field GF(2).

Definition 1. For $\alpha=\left(\alpha_{1}, \ldots, \alpha_{n}\right)$ and $x=\left(x_{1}, \ldots, x_{n}\right) \in\{0,1\}^{n}$, we define $m_{\alpha}(x):=\prod_{i} x_{i}^{\alpha_{i}}$ and the degree $\operatorname{deg} m_{\alpha}:=|\alpha|:=\#\left\{i \mid \alpha_{i}=1\right\}$.

Theorem 2. (Algebraic Normal Form) Let $f:\{0,1\}^{n} \rightarrow\{0,1\}$ be a Boolean function. Then, $f(x)$ can be written as $f(x)=\bigoplus_{\alpha \in\{0,1\}^{n}} c_{\alpha} \cdot m_{\alpha}(x)$ for unique coefficients $c_{\alpha} \in\{0,1\}$. Hence, the value $\max \left\{\operatorname{deg} m_{\alpha} \mid c_{\alpha} \neq 0\right\}$ is unique and is called the degree $\operatorname{deg} f$ of $f$.

Definition 3. For $\alpha=\left(\alpha_{1}, \ldots, \alpha_{n}\right), \alpha^{\prime}=\left(\alpha_{1}^{\prime}, \ldots, \alpha_{n}^{\prime}\right) \in\{0,1\}^{n}$ we say that $\alpha^{\prime} \leq \alpha$ if $\forall i: \alpha_{i}^{\prime} \leq \alpha_{i}$ (treated as integers). Consequently, we say that $\alpha^{\prime}<\alpha$ if $\alpha^{\prime} \leq \alpha$ but $\alpha^{\prime} \neq \alpha$. For $\alpha^{\prime} \leq \alpha$ we define $\alpha-\alpha^{\prime}:=\left(\alpha_{1}-\alpha_{1}^{\prime}, \ldots, \alpha_{n}-\alpha_{n}^{\prime}\right)$.

Obviously, $\alpha^{\prime} \leq \alpha$ (resp. $\alpha^{\prime}<\alpha$ ) implies $\operatorname{deg} m_{\alpha^{\prime}} \leq \operatorname{deg} m_{\alpha}$ (resp. $\operatorname{deg} m_{\alpha^{\prime}}<$ $\left.\operatorname{deg} m_{\alpha}\right)$.

Lemma 4. Let $\alpha, \delta^{(1)}, \delta^{(2)} \in\{0,1\}^{n}$ be arbitrary. For $i=1,2$ it holds that $m_{\alpha}\left(x \oplus \delta^{(i)}\right)=\bigoplus_{\alpha^{\prime} \leq \alpha} m_{\alpha^{\prime}}(x) m_{\alpha-\alpha^{\prime}}\left(\delta^{(i)}\right)$ and $m_{\alpha}\left(x \oplus \delta^{(1)}\right) \oplus m_{\alpha}\left(x \oplus \delta^{(2)}\right)$ has a degree $\leq \operatorname{deg} m_{\alpha}-1$. 
Proof. The first equation is obvious. The second one is because of:

$m_{\alpha}\left(x+\delta^{(1)}\right)+m_{\alpha}\left(x+\delta^{(2)}\right)$

$=\sum_{\alpha^{\prime} \leq \alpha}\left(m_{\alpha^{\prime}}(x) m_{\alpha-\alpha^{\prime}}\left(\delta^{(1)}\right)+m_{\alpha^{\prime}}(x) m_{\alpha-\alpha^{\prime}}\left(\delta^{(2)}\right)\right)$

$=\underbrace{m_{\alpha}(x)+m_{\alpha}(x)}_{=0}+\sum_{\alpha^{\prime}<\alpha}\left(m_{\alpha^{\prime}}(x) m_{\alpha-\alpha^{\prime}}\left(\delta^{(1)}\right)+m_{\alpha^{\prime}}(x) m_{\alpha-\alpha^{\prime}}\left(\delta^{(2)}\right)\right)$.

Theorem 5. Let $f$ be a Boolean function with $\operatorname{deg} f=d$. Then, $f\left(x \oplus \delta^{(1)}\right) \oplus$ $f\left(x \oplus \delta^{(2)}\right)$ has a degree $\leq d-1$.

Proof. By Theorem 2 we can write $f(x)$ as $\sum_{\alpha,|\alpha| \leq d} c_{\alpha} m_{\alpha}(x)$. Then, by lemma 4 it is $f\left(x \oplus \delta^{(1)}\right) \oplus f\left(x \oplus \delta^{(2)}\right)=\bigoplus_{\alpha,|\alpha| \leq d} c_{\alpha} \underbrace{\left(m_{\alpha}\left(x \oplus \delta^{(1)}\right) \oplus m_{\alpha}\left(x \oplus \delta^{(2)}\right)\right)}_{\text {deg } \leq d-1}$.

The following corollary is obvious:

Corollary 6. For any even number $m$ and any vectors $\delta^{(1)}, \ldots, \delta^{(m)}$, the degree of the function $\bigoplus_{i=1}^{m} f\left(x \oplus \delta^{(i)}\right)$ is $\leq \operatorname{deg} f-1$.

Theorem 7 is a special case of theorem 5 and will be of use in this paper:

Theorem 7. Let $f$ be a Boolean function with $\operatorname{deg} f=d$. Let $\boldsymbol{e}_{i} \in\{0,1\}^{n}$ be the unit vector with its only 1 in position $i$. Then the function $f_{1}\left(x^{\prime}\right)=f(x) \oplus f\left(x \oplus \boldsymbol{e}_{i}\right)$ has degree $\leq d-1$, where $x^{\prime}=\left(x_{1}, \ldots, x_{i-1}, x_{i+1}, \ldots, x_{n}\right) \in\{0,1\}^{n-1}$.

Proof. We first split the function $f(x)$ into two parts, the first part consisting of the monomials containing $x_{i}$, and the second part consisting of the monomials not containing $x_{i}$ as a factor:

$$
f(x)=x_{i} \cdot f_{1}\left(x^{\prime}\right) \oplus f_{2}\left(x^{\prime}\right),
$$

where it is straightforward to see that $\operatorname{deg} f_{1} \leq d-1$ and $\operatorname{deg} f_{2} \leq d$. We do the same for the function $f\left(x \oplus \boldsymbol{e}_{i}\right)$ :

$$
f\left(x \oplus \boldsymbol{e}_{i}\right)=\left(x_{i} \oplus 1\right) \cdot f_{1}\left(x^{\prime}\right) \oplus f_{2}\left(x^{\prime}\right) .
$$

Taking the XOR of the equations (11) and (2) and eliminating terms occurring twice yields: $f(x) \oplus f\left(x \oplus \boldsymbol{e}_{i}\right)=f_{1}\left(x^{\prime}\right)$.

\subsection{General Framework for Synchronous Stream Ciphers}

We consider a synchronous stream cipher with $n$-bit state $S$ updated by a linear function represented by a matrix $L$ (e.g., one or more LFSRs) over $\mathbb{Z}_{2}$, and with a nonlinear output function $f$ that takes $\varphi$ input bits coming from $S$ to produce one output bit $z_{t}$. Some designs (e.g., the combiners with memory) also include a $m$-bit memory $M$ that has a nonlinear update function $h$. This results in the following general framework for a synchronous stream cipher:

$$
\left\{\begin{array}{l}
z_{t}=f\left(S_{t}, M_{t}\right) \\
c_{t}=p_{t} \oplus z_{t} \\
S_{t+1}=L \cdot S_{t} \\
M_{t+1}=h\left(S_{t}, M_{t}\right),
\end{array}\right.
$$


where $p_{t}, z_{t}$ and $c_{t}$ are respectively the plaintext, the key stream and the ciphertext at time $t=0,1,2 \ldots$

The initial state $\left(S_{0}, M_{0}\right)$ is determined by the resynchronization mechanism, which combines a $k$-bit secret key $K$ and a $\iota$-bit known initialization vector $I V^{i}$ with an initialization function $f_{\text {init }}$, i.e., $\left(S_{0}, M_{0}\right)=f_{\text {init }}\left(K, I V^{i}\right)$.

\subsection{Algebraic Attacks}

In this section we repeat some facts about algebraic attacks against LFSR-based key stream generators. We describe the general attack on combiners with memory introduced in [3] as this includes the special case of memoryless combiners 8 .

An algebraic attack works as follows: first find a Boolean function $F \not \equiv 0$ (called an ad-hoc equation) such that for all $t$

$$
0=F\left(L^{t} \cdot K, \ldots, L^{t+r-1} \cdot K, z_{t}, \ldots, z_{t+r-1}\right) .
$$

Such a function $F$ can be found with the algorithm of $[3$ if $\varphi \cdot r$ is not too large.

Secondly, recover the secret key $K$ by solving this system of equations. For this purpose, several methods (Linearization, XL, XSL, Groebner bases algorithms such as F4 and F5,...) exist. Amongst them only the linearization method allows a general estimation of the work effort. We give now a description of the linearization method. Due to the linearity of $L$, all equations (44) have degree $\leq d:=\operatorname{deg} F$. Therefore, the number $\mathcal{M}$ of different monomials occurring is upper bounded by $\beta(k, d):=\left(\begin{array}{l}k \\ 0\end{array}\right)+\ldots+\left(\begin{array}{l}k \\ d\end{array}\right)$.

By replacing each monomial by a new variable, the attacker gets a linear system of equations in $\mathcal{M}$ unknowns. It can be solved by Gaussian elimination or more refined methods like the one by Strassen [27. As $\beta(k, d) \in O\left(k^{d}\right)$, the lower the degree $d$, the faster the attack.

\subsection{Resynchronization Attacks}

For a synchronous stream cipher, perfect synchronization between sender and receiver is required. The aim of the resynchronization mechanism is to achieve this in a secure fashion.

A first solution is fixed resync. In this scenario, the message is divided into frames, and each frame $i$ is encrypted with a unique $I V^{i}$, a frame counter updated in a deterministic way. An attack under this scenario is called a known IV resynchronization attack. The frequency at which resynchronization should occur depends on the risk of synchronization loss. Examples of stream ciphers that use fixed resync are the $E 0$ algorithm used in the Bluetooth [6] wireless communication standard, and A5 [1] used in GSM cellular voice and data communication.

A second scenario is that the receiver sends a resynchronization request to the sender as soon as synchronization loss is detected. This is called requested resync. In this scenario, the receiver may be allowed to choose the nonce $I V^{i}$ used in the frame. This may enable a chosen $I V$ resynchronization attack, as described e.g. by Joux and Muller [16. Security under the chosen $I V$ attack scenario implies 
security under the known $I V$ attack scenario. Hence, a good resynchronization mechanism should be resistant against a chosen $I V$ attack.

We now describe a first resynchronization attack by Daemen et al. [10] on a simplified version of this framework and point out its limitations.

\section{The Daemen et al. Resynchronization Attack}

\subsection{Description}

The resynchronization attack of Daemen et al. is a known plaintext attack for the special case of a simple memoryless combiner with a linear resynchronization mechanism.

The framework of the attack can be described as follows:

$$
\left\{\begin{array}{l}
S_{0}^{i}=A \cdot K \oplus B \cdot I V^{i} \\
z_{t}^{i}=f\left(\Pi \cdot S_{t}^{i}\right) \\
S_{t+1}^{i}=L \cdot S_{t}^{i} .
\end{array}\right.
$$

In these equations, $A, B, L$ and $\Pi$ are known binary matrices, $A \in \mathbb{Z}_{2}^{n \times k}, B \in$ $\mathbb{Z}_{2}^{n \times \iota}, L \in \mathbb{Z}_{2}^{n \times n}$ and $\Pi \in \mathbb{Z}_{2}^{\varphi \times n}$. The matrices $A$ and $B$ represent the fact that the resync mechanism is linear, the projection matrix $\Pi$ shows that the output function $f$ uses only a subset of $\varphi$ bits of $S_{t}^{i}$. The initialization vector of the $i$ th frame is $I V^{i}$, and $z_{t}^{i}$ is the key stream bit at time $t$ of the $i$ th frame.

We introduce the key-dependent unknown values $\mathrm{K}_{t}$ and the known values $\mathrm{IV}_{t}^{i}$ as follows:

$$
\left\{\begin{array}{l}
\mathrm{K}_{t}=\Pi \cdot L^{t} \cdot A \cdot K \\
\mathrm{IV}_{t}^{i}=\Pi \cdot L^{t} \cdot B \cdot I V^{i}
\end{array}\right.
$$

The attacker can set up a system of equations built as follows:

$$
z_{t}^{i}=f\left(\mathrm{~K}_{t} \oplus \mathrm{IV}_{t}^{i}\right) \quad \text { for } 0 \leq i \leq R-1,0 \leq t \leq T-1,
$$

where $R$ is the number of resynchronizations and $T$ is the number of key stream bits we know in each frame. We try to find a solution of this system of equations for each $t$. Assume without loss of generality that $t=0$. If $\varphi$ is not too large, we can perform an exhaustive search over $\mathrm{K}_{0}$, and check whether the guess satisfies the $R$ equations for $t=0$. If $R \geq \varphi$, it is expected that a unique solution for $\mathrm{K}_{0}$ exists. Hence the correct value of $\mathrm{K}_{0}$ has been found, and thus $\varphi$ linear equations in the bits of the secret key. This is repeated for $t=1,2, \ldots, p-1$, such that $p=\lceil k / \varphi\rceil$, until the entire secret key is deduced.

The complete attack requires on average $\lceil k / \varphi\rceil \cdot 2^{\varphi}$ evaluations of the function $f$, at least $\varphi$ resyncs and about $k$ bits of key stream in total ( $\varphi$ frames of length $\lceil k / \varphi\rceil)$. Note that the computational complexity of the attack increases exponentially with the number $\varphi$ of inputs of the Boolean function $f$.

\subsection{Limitations}

The Daemen et al. attack can be seen as a divide-and-conquer attack. Standard cryptanalytic attacks such as correlation and algebraic attacks work chronologically on a key stream, which corresponds to the output of one frame. On the 
contrary, the Daemen et al. attack tries to solve the system by working on one specific time over all frames. One of the motivations of the paper is to combine both approaches. Of special interest is the combination of the resynchronization attack with algebraic attacks.

In many cases, it is not obvious whether the approach by Daemen et al. works or not. We have identified the following limitations:

1. The attack does not work in real time.

2. The number of resyncs $R$ has to be at least the number $\varphi$ of input bits of the output function $f$.

3. The complexity is prohibitively large for large values of $\varphi$.

4. The divide-and-conquer approach does not work if the key stream generator uses additional memory.

5. The initialization function $f_{\text {init }}$ has to be linear.

In this paper, we will address each of these limitations and show how to overcome them.

\section{Real-Time Attack}

The attack of Daemen et al. shows that ciphers that use a linear resynchronization mechanism and that have a Boolean function $f$ with few inputs are insecure. This enables a passive attack on such designs. A real-time attack in which the attacker can discover the plaintext immediately (and even modify it in a controlled way) is not possible, because the time required to perform the $p$ exhaustive searches will be too high. Here we show how to easily replace this iterated exhaustive search by a precomputation step. This enables real-time active attacks on such ciphers.

We start from the realistic assumption that the $I V^{i}$ s are chosen in a deterministic way, for instance by a counter or a fixed update mechanism. In the precomputation step, we first calculate the values $\operatorname{IV}_{t}^{i}$ for $0 \leq i \leq \varphi-1$ and $0 \leq t \leq T-1$. Then we calculate the following $\varphi$ bits, and repeat this for all values of $\mathrm{G}_{t}$ (a guess for $\mathrm{K}_{t}$ ) going from 0 to $2^{\varphi}-1$, and this for all $t$.

$$
\left\{\begin{array}{l}
f\left(\mathrm{G}_{t} \oplus \mathrm{IV}_{t}^{0}\right)=b_{\mathrm{G}_{t}, t}^{0} \\
f\left(\mathrm{G}_{t} \oplus \mathrm{IV}_{t}^{1}\right)=b_{\mathrm{G}_{t}, t}^{1} \\
\cdots \\
f\left(\mathrm{G}_{t} \oplus \mathrm{IV}_{t}^{\varphi-1}\right)=b_{\mathrm{G}_{t}, t}^{\varphi-1} .
\end{array}\right.
$$

For each time $t$, we obtain $2^{\varphi}$ sequences $b_{\mathrm{G}_{t}, t}^{0} b_{\mathrm{G}_{t}, t}^{1} \ldots b_{\mathrm{G}_{t}, t}^{\varphi-1}$. Because the length of these sequences is $\varphi$, every value of $\mathrm{G}_{t}$ is expected to correspond with a unique sequence $b_{\mathrm{G}_{t}, t}^{0} b_{\mathrm{G}_{t}, t}^{1} \ldots b_{\mathrm{G}_{t}, t}^{\varphi-1}$. We then sort the $\mathrm{G}_{t}$ values based on the numerical value of the corresponding sequence, and store this in memory.

The attack now goes as follows. We group the outputs observed at say $t=0$ in a sequence $z_{0}^{0} z_{0}^{1} \ldots z_{0}^{\varphi-1}$. We jump to this position in our table built for $t=0$, and the value found there is the correct value of $\mathrm{K}_{0}$. We do the same for the times $t=1,2, \ldots p-1$, and we have then found the necessary $\mathrm{K}_{i}$ to directly determine the secret key $K$. 
The total complexity of the precomputation step is about $k \cdot 2^{\varphi}$ evaluations of $f$ (but this can of course be replaced by $2^{\varphi}$ evaluations of $f$ and $k \cdot 2^{\varphi}$ table look-ups). The memory requirement is about $k \cdot 2^{\varphi}$ bits, which is feasible for many stream ciphers (e.g., for a secret key of $k=256 \mathrm{bits}$, and a Boolean function with $\varphi=20$ inputs, 32 Mbyte is required).

\section{$5 \quad$ Attack with a Small Number of Resynchronizations}

In [10], the authors made the assumption that the number of solutions converges to 1 if $R \gtrsim \varphi$. Actually, the number of required resyncs depends on the cipher and the observed public parameters $I V^{i}$. In [15, Golic and Morgari discussed the number of $I V$ s that are needed for the Daemen et al. attack to work. They showed that with a non-negligible probability more than $\varphi$ known $I V$ s are necessary. This results in an increased attack complexity, both for the original attack and for the precomputation attack.

We will here follow different approaches. We want the attacks to work in any case and with a minimal number of known $I V$ s. Simulations on various Boolean functions have confirmed that the standard resynchronization attack does not always work in practice with $\varphi I V \mathrm{~s}$. This is due to two reasons, the first being imperfect behavior of the function $f$. However, this effect is not very important because in most stream ciphers the function $f$ has good statistical properties, which typically include balancedness and high nonlinearity. A second reason is that sometimes collisions occur between two values $\operatorname{IV}_{t}^{a}$ and $\operatorname{IV}_{t}^{b}$ where $a \neq b$. We will show several ways to overcome this problem.

\subsection{Computational Approach}

Two-Phase Attack. We implement the algorithm in two steps. The first step, the resynchronization attack, retains a set of values for each of the $\mathrm{K}_{0}, \mathrm{~K}_{1} \ldots \mathrm{K}_{p-1}$. In a second step, we then search through all possible combinations until we have found the correct secret key.

Simulations have shown that for $\varphi$ (or more) known $I V \mathrm{~s}$, the time complexity of the second step is negligible. In other words, the resynchronization attack (extended with the fast search step) is always successful under realistic assumptions with $\varphi$ known $I V$ s.

Using this two-step algorithm, one can also mount a resynchronization attack with $R<\varphi$ known $I V$ s. The time complexity of the second step can then be shown to be about $2^{(\varphi-R) \cdot \frac{k}{\varphi}}$. Even if this complexity increases exponentially with decreasing $R$, this shows that a resynchronization attack is still feasible for $R$ smaller than (but close to) $\varphi$.

Overlapping Bits. There is also another interesting way to perform a resynchronization attack when $R<\varphi$. Let's take the case $R=\varphi-1$. For $\mathrm{K}_{t}$, we will get two possibilities after the exhaustive search. But looking at the bits of these two possibilities $\mathrm{K}_{t, 1}$ and $\mathrm{K}_{t, 2}$, about half of these will be equal, and will therefore 
certainly be the correct values for these bits of $\mathrm{K}_{t}$. This implies that we have still found $\varphi / 2$ linear equations in $K$, and we will just need frames that are twice as long as in the standard attack, i.e., have length $T \geq 2 k / \varphi$ each. This is still very realistic in most cases. We can develop a similar reasoning for smaller values of $R$, but the length of the frames and the complexity required increases rapidly: they can be shown to be $2^{2^{\varphi-R}-1} \cdot k / \varphi$ and $2^{2^{\varphi-R}-1} \cdot k / \varphi \cdot 2^{\varphi}$ respectively (see the extended version of this paper [2]).

\subsection{Using Algebraic Attacks}

The resync scenario implies the following system of equations:

$$
z_{t}^{i}=f\left(\mathrm{~K}_{t} \oplus \mathrm{IV}_{t}^{i}\right), \quad 0 \leq i \leq R-1,0 \leq t \leq T-1 .
$$

Hence, another possibility is to try to solve it as a whole instead of working time per time. The linearization method described in Sect. 2.3 requires that the number of linearly independent equations exceeds the number $\mathcal{M}$ of occurring monomials. This requires $T \cdot R \geq \mathcal{M}$. As $\mathcal{M}$ is upper bounded by $\beta\left(k, d^{\prime}\right) \in O\left(k^{d^{\prime}}\right)$ with $d^{\prime}=\operatorname{deg} f$, the lower the degree of the equations the faster the attack. In the literature [8, 9, 19, 5, several conditions and methods are described for transforming (9) into a new system of equations

$$
g\left(\mathrm{~K}_{t} \oplus \mathrm{IV}_{t}^{i}, z_{t}^{i}\right)=0, \quad 0 \leq i \leq R-1,0 \leq t \leq T-1 .
$$

with $d:=\operatorname{deg} g<\operatorname{deg} f$. Next, we will show how to use the resync setting to decrease the degree of (10) further.

The Degree- $\boldsymbol{d}$-1 Attack. The first approach is to construct new equations of degree $\leq d-1$. We express $g$ by

$$
g\left(\mathrm{~K}_{t} \oplus \mathrm{IV}_{t}^{i}, z_{t}^{i}\right)=\bigoplus_{j} g_{j}\left(\mathrm{~K}_{t} \oplus \mathrm{IV}_{t}^{i}\right) \cdot \tilde{g}_{j}\left(z_{t}^{i}\right) .
$$

Observe that the functions $g_{j}$ and $\tilde{g}_{j}$ depend only on $g$ and are all known to the attacker. The idea is to find appropriate linear combinations of (11) to reduce the degree. Let $I:=\left\{j \mid \operatorname{deg} g_{j}=d\right\}$ and rewrite (11) to

$$
g=\underbrace{\bigoplus_{j \in I} g_{j} \cdot \tilde{g}_{j}}_{\operatorname{deg} g_{j}=d} \oplus \underbrace{\bigoplus_{j \notin I} g_{j} \cdot \tilde{g}_{j}}_{\operatorname{deg} g_{j}<d} .
$$

Theorem 8 provides a method for decreasing the degree at least by 1 :

Theorem 8. Let $g$ be expressed as described in (11). For any known $\mathrm{IV}_{t}^{0}, \ldots, \mathrm{IV}_{t}^{|I|}$ and corresponding known outputs $z_{t}^{i}$, coefficients $c_{0}, \ldots, c_{|I|} \in\{0,1\}$ with at least one $c_{i} \neq 0$ can be computed such that the degree of $\bigoplus_{i=0}^{|I|} c_{i} \cdot g\left(\mathrm{~K}_{t} \oplus \mathrm{IV}_{t}^{i}, z_{t}^{(i)}\right)$ is $\leq \operatorname{deg} g-1$. 
Proof. We set $g_{j}^{i}:=g_{j}\left(\mathrm{~K}_{t} \oplus \mathrm{IV}_{t}^{i}\right)$ and $\tilde{g}_{j}^{i}:=\tilde{g}_{j}\left(z_{t}^{i}\right) \in\{0,1\}$. With (11), we can write

$$
\bigoplus_{i=0}^{|I|} c_{i} \cdot g\left(\mathrm{~K}_{t} \oplus \mathrm{IV}_{t}^{i}, z_{t}^{i}\right)=\bigoplus_{j \in I} \bigoplus_{i=0}^{|I|} c_{i} \cdot \tilde{g}_{j}^{i} \cdot g_{j}^{i} \oplus \bigoplus_{j \notin I} \bigoplus_{i=0}^{|I|} c_{i} \cdot \tilde{g}_{j}^{i} \cdot g_{j}^{i} .
$$

The second part of the right hand side has a degree $\leq d-1$ by definition of $I$. The idea is to find coefficients $c_{0}, \ldots, c_{|I|} \in\{0,1\}$ such that the first part of the right hand side has degree $\leq d-1$ too. By Corollary 6, it is sufficient that $\sum_{i=0}^{|I|} c_{i} \cdot \tilde{g}_{j}^{i}$ (treated as an integer) is an even number for all $j \in I$.

We show now that it is always possible. For each $i$ we define the vector $\vec{V}_{i}:=$ $\left(\tilde{g}_{1}^{(i)}, \ldots, \tilde{g}_{|I|}^{(i)}\right) \in\{0,1\}^{|I|}$. Then the assumption above is equivalent to $\bigoplus_{i} c_{i} \cdot \vec{V}_{i}=$ $\overrightarrow{0}$. By the theory of linear algebra, the $|I|+1$ vectors of the $|I|$-dimensional vector space $\{0,1\}^{|I|}$ are linearly dependent. Therefore, such coefficients $c_{i}$ exist.

Let $\mathcal{M}_{e}$ be the number of monomials of degree $\leq e$ occurring in (10). The attack complexity is as follows. First we have to calculate (for a fixed clock $t$ ) the coefficients $c_{i}$. This requires $O\left(|I|^{3}\right)$ operations. Then, the computation of the function of degree $\leq d-1$ is equivalent to the summation of (several) vectors of size $\mathcal{M}_{d-1}$. The two steps have to be repeated about $\mathcal{M}_{d-1}$ times to get enough linearly independent equations of degree $\leq d-1$. The final step is to use Gaussian elimination to solve the linearized system of equations $\approx\left(\mathcal{M}_{d-1}\right)^{3}$. Therefore the overall number of operations is about $\left(|I|^{3}+M_{d-1}\right) \cdot M_{d-1}+\left(M_{d-1}\right)^{3}$. Because of $\mathcal{M}_{e} \leq \beta(k, e)$, an upper bound is $\left(|I|^{3}+\beta(k, d-1)\right) \cdot \beta(k, d-1)+\beta(k, d-1)^{3}$. Note that it may happen that $\bigoplus_{i} c_{i} \cdot g\left(\mathrm{~K}_{t} \oplus \mathrm{IV}_{t}^{i}, z_{t}^{i}\right)$ is equal to zero for some $t 1$

As opposed to fast algebraic attacks 9, 4, this approach does not require the highest-degree monomials to be independent of the key stream bits. Moreover, the number of key stream bits required is $\leq \beta(k, d-1)+|I|$ instead of $\leq \beta(k, d)$. On the other hand, fast algebraic attacks benefit from the fact that the most time consuming part can be sourced out in a precomputation step. This is not possible here. Another advantage is that its applicability is independent of the values of $\mathrm{IV}_{t}^{i}$ and $z_{t}^{i}$ and that it does not require $\varphi$ to be low.

The Degree- $\boldsymbol{e}$ Attack. So far, we concentrated only on decreasing the degree by 1 . But clever combinations may reduce the degree even further. In the worst case these combinations may be linear, even if the degree of $g$ is high. This is for example the case for the $E_{0}$ key stream generator We develop now the theory how to compute the lowest possible degree. In the following, we treat $\mathrm{K}$ as $\varphi$ unknowns.

Definition 9. We set $S(g):=\left\{g(\mathrm{~K} \oplus \mathrm{IV}, z) \mid \mathrm{IV} \in\{0,1\}^{\varphi}, z \in\{0,1\}\right\}$ and define by $<g>:=<S(g)>$ the linear span of $S(g)$ (i.e., all possible linear combina-

\footnotetext{
${ }^{1}$ For example, this cannot be avoided if $g$ is linear. But in this case, the cipher is weak anyhow.

${ }^{2}$ The best before was a system of equations of degree 3 (see 9 ).
} 
tions). $\langle g\rangle$ is a vector space over the finite field GF(2). By dim $g$ we define the dimension of $\langle g\rangle$ and by $B(g)$ an arbitrary basis of $\langle g\rangle$. Further on we set $\mathcal{M}_{d}(g)$ to be all monomials of degree $\leq d$ which occur in $S(g)$.

From the theory of linear algebra, the following theorem is obvious:

Theorem 10. A function of degree $\leq e$ exists in $\langle g\rangle$ only if the vectors in $B(g) \cup \mathcal{M}_{e}(g)$ are linearly dependent.

Let $\mathcal{S}$ be a set of Boolean functions. We now describe an algorithm to compute a linearly independent set of functions $\langle\mathcal{S}\rangle$ with the lowest possible degree $e$ : We treat the functions in $\mathcal{S}$ as rows of a matrix where each column reflects one occuring monomial. Then, we apply Gaussian elimination in such way that the monomials with the highest degree are eliminated first and so on. Finally, we just pick those functions in the result with the lowest degree.

If $\mathcal{S}=B(g)$, the algorithm computes the lowest possible value for $e$. Let $\tilde{B}:=\left\{g\left(\mathrm{~K}_{t} \oplus \mathrm{IV}_{t}^{i}, z_{t}^{i}\right) \mid 0 \leq i \leq R-1\right\}$ be the set of functions available to the attacker. $\langle\tilde{B}\rangle$ might be only a subset of $\langle g\rangle$. In this case, the lowest possible degree can be higher.

We try now to estimate the complexity of the Degree- $e$ attack. The first step is to find an appropriate linear combination in $\tilde{B}$. The effort is about $(\operatorname{dim} g)^{2} \cdot\left|\mathcal{M}_{d}(g)\right|$ to find the linear combination and about $\mathcal{M}_{e}$ to compute the corresponding vector of size $\mathcal{M}_{e}$. This has to be repeated at least $\mathcal{M}_{e}$ times. Finally, a system of equations in $\mathcal{M}_{e}$ has to be solved $\left(\approx \mathcal{M}_{e}^{3}\right)$. Hence, the overall number of operations is about $\left((\operatorname{dim} g)^{2} \cdot\left|\mathcal{M}_{d}(g)\right|+\mathcal{M}_{e}\right) \cdot \mathcal{M}_{e}+\mathcal{M}_{e}^{3}$. Because of $\mathcal{M}_{e} \leq \beta(k, e)$ and $\operatorname{dim} g \leq \mathcal{M}_{d} \leq \beta(k, d)$, the following expression is an upper bound for the complexity $\left(\beta(\varphi, d)^{3}+\beta(k, d)\right) \cdot \beta(k, e)+\beta(k, e)^{3}$.

In the individual case, the applicability of this attack depends on many parameters: the function $g$, the number $R$ of accessible frames and the corresponding values of $\mathrm{IV}^{i}$ and $z_{t}^{i}$. Hence the attack does not work in every case. On the other hand, it puts on the designer the responsibility of making sure that these attacks are not feasible.

Moreover, if the set $\tilde{B}$ is a basis of $\langle g\rangle$, then an equation of the lowest possible degree can be constructed. What is the probability that this happens? Let $s:=\operatorname{dim} g$ and $R \geq s$. If we assume that each expression $g\left(\mathrm{~K}_{t} \oplus \mathrm{IV}_{t}^{i}, z_{t}^{i}\right)$ is a random vector in $\{0,1\}^{s}$ then by [30], the probability that $\tilde{B}$ is a basis of $\langle g\rangle$ is Prob $=\prod_{i=R-s+1}^{m}\left(1-1 / 2^{i}\right)$.

\section{Resynchronization Attacks with Large $\varphi$}

The Daemen et al. attack only works when the number $\varphi$ of inputs to the Boolean function is not too large. However, we will show in this section that using a linear resynchronization mechanism will inevitably induce weaknesses into stream ciphers, even when $\varphi$ is very large. We will show a chosen $I V$ attack, a known $I V$ attack and an algebraic attack. 


\subsection{A Chosen IV Attack}

The standard attack has a large time complexity of $\lceil n / \varphi\rceil \cdot 2^{\varphi}$ evaluations of the function $f$, but it only requires $\varphi$ resyncs. We will now show that a tradeoff is possible.

Let $\mathrm{K}_{t}$ in (7) consist of the bits $k_{0}, k_{1}, \ldots k_{\varphi-1}$. We make the reasonable assumption that in the chosen $I V$ attack, the attacker can control the values of $\mathrm{IV}_{t}^{i}$, consisting of the bits $i v_{0}, i v_{1}, \ldots i v_{\varphi-1}$. We now start the chosen $I V$ attack. We first take a constant $C$. We then perform resyncs with all the values $\operatorname{IV}_{t}^{i}=C \oplus i$, where we let $i$ take all values 3 going from $0(00 \ldots 0)$ to $2^{u}-1(00 \ldots 011 \ldots 1)$ for some $u$. Let's consider the first two values of our resynchronization attack. We denote $k_{i} \oplus i v_{i}$ as $x_{i}$. We know that:

$$
\left\{\begin{array}{l}
f\left(x_{0}, x_{1}, \ldots x_{\varphi-1}\right)=z_{0}^{0} \\
f\left(x_{0}, x_{1}, \ldots x_{\varphi-1} \oplus 1\right)=z_{0}^{1} .
\end{array}\right.
$$

By XORing both equations and using Theorem 7 we get:

$$
f_{1}\left(x_{0}, x_{1}, \ldots x_{\varphi-2}\right)=z_{0}^{0} \oplus z_{0}^{1},
$$

where the Boolean function $f_{1}$ has many properties that are desirable for an attacker. The degree of $f_{1}$ is lower, it has fewer monomials and it depends on less variables than $f$. This makes many attacks much easier.

In our attack, we will apply this method with $2^{u}$ chosen $I V \mathrm{~s}$ in an iterative way. As an illustration, these are the equations for $u=2$.

$$
\left.\left.\begin{array}{l}
f\left(\ldots x_{\varphi-2}, x_{\varphi-1}\right)=z_{0}^{0} \\
f\left(\ldots x_{\varphi-2}, x_{\varphi-1} \oplus 1\right)=z_{0}^{1} \\
f\left(\ldots x_{\varphi-2} \oplus 1, x_{\varphi-1}\right)=z_{0}^{2} \\
f\left(\ldots x_{\varphi-2} \oplus 1, x_{\varphi-1} \oplus 1\right)=z_{0}^{3}
\end{array}\right\} \Rightarrow \begin{array}{l}
f_{1}\left(\ldots x_{\varphi-2}\right)= \\
z_{0}^{0} \oplus z_{0}^{1} \\
f_{1}\left(\ldots x_{\varphi-2} \oplus 1\right)= \\
z_{1}^{2} \oplus z_{0}^{3}
\end{array}\right\} \Rightarrow \begin{aligned}
& f_{2}\left(x_{0} \ldots x_{\varphi-3}\right)= \\
& z_{0}^{0} \oplus z_{0}^{1} \oplus z_{0}^{2} \oplus z_{0}^{3}
\end{aligned}
$$

The basic attack requires at every time $2^{u} \cdot \varphi$ resyncs, in order to obtain $\varphi-u$ equations in the Boolean function $f_{u}\left(x_{0} \ldots x_{\varphi-u-1}\right)$ which can then be used in a normal resynchronization attack.

In practice, however, we note that the number of monomials, variables and the degree of the equation decreases very rapidly, making the attack work with very small complexity.

\subsection{A Known $I V$ Attack}

We now describe another attack, which shows that the linear resynchronization mechanisms introduces weaknesses in the fixed resync setting for all Boolean functions.

${ }^{3}$ The impact of this choice of $i$ is that the last $u$ input bits of $f$ will take all possible values. Of course we can do the same with any combination of $u$ bits by choosing $i$ as needed. 
The principle of the attack is similar to the linear cryptanalysis method, developed by Matsui for attacking block ciphers [18. First we search for a linear expression for the Boolean function that holds with probability $p \neq 0.5$. We then collect sufficiently many resyncs such that we can determine key bits using a maximum likelihood method. We will now describe this in more detail.

Our starting point is the fact that for any $\varphi$-input nonlinear Boolean function $f$, we can always find a subset $S \subset\{0,1, \ldots \varphi-1\}$ for which the equation

$$
\bigoplus_{i \in S} x_{i}=f\left(x_{0}, \ldots x_{\varphi-1}\right)
$$

holds with probability $0.5+\epsilon$, where $\epsilon \neq 0$. Suppose that the best bias we have found is $\epsilon(0<\epsilon \leq 0.5)$. For each time $t$, with $R$ known $I V \mathrm{~s}$, we get the following equations:

$$
\left\{\begin{array}{l}
\bigoplus_{i \in S} k_{i}=\bigoplus_{i \in S} i v_{i}^{0} \oplus z_{t}^{1} \\
\vdots \\
\bigoplus_{i \in S} k_{i}=\bigoplus_{i \in S} i v_{i}^{I-1} \oplus z_{t}^{I-1},
\end{array}\right.
$$

each of which holds with probability $0.5+\epsilon$. We now count for how many of these equations the right hand side is 1 respectively 0 . We assume then that the correct right hand side is the value ( 0 or 1 ) that occurs most if $\epsilon>0$, and the value that occurs least if $\epsilon<0$.

We now have found one linear equation in the state bits that is true with some probability. This probability increases with the value of $R$ and is dependent on the magnitude of $\epsilon$. As in [18], the probability that the equation is correct, given $R$ resyncs and a bias $\epsilon$, is equal to:

$$
\int_{-2 \cdot \sqrt{R} \cdot|\epsilon|}^{\infty} \frac{1}{\sqrt{2 \cdot \pi}} \cdot e^{-x^{2} / 2} \cdot d x=0.5+0.5 \cdot \operatorname{erf}(\sqrt{2 \cdot R} \cdot|\epsilon|),
$$

where erf is the error function. If we want the probability of correctness to approach one, we need the number of resyncs $R$ to be $c \cdot \epsilon^{-2}$ for a small constant value $c$.

The output of a Boolean function is correlated to at least one linear function of the inputs, see Xiao and Massey [29. The smallest bias $\epsilon$ that can be found 4 is at least $2^{-\frac{\varphi}{2}-1}$. This implies that any linear resynchronization mechanism with a $\varphi$-input Boolean function $f$ can be broken by this resynchronization attack using at most about $2^{\varphi+2}$ known $I V$ s.

How to search for the best linear approximation has been well-studied. The Walsh-Hadamard transform can be used to find the best linear approximation,

\footnotetext{
${ }^{4}$ This lower bound for $\epsilon$ follows from the universal nonlinearity bound for Boolean functions. Equality applies to the so-called bent functions. Stream ciphers typically do not use bent functions because they are not balanced. The size of the smallest bias to be found in balanced Boolean functions is still an open problem, but some bounds have been presented, see 12 for an overview. For simplicity, we take the bias of bent functions, but the bias for actual functions will be higher and therefore less resyncs will be needed in practice.
} 
see 24] for a thorough treatment. In the context of correlation attacks, such linear approximations have been studied in the literature, both for memoryless combiners and nonlinear filter generators 24 as for combiners with memory [13, 20 .

The biases found in actual Boolean functions used in stream ciphers will be much higher than the lower bounds described above. This is due to several reasons: the functions have to be balanced, they have to be easily implementable, and for combiners they will also have to take into account the trade-off that has to be made between nonlinearity and resilience, see Sarkar and Maitra 26. It can be expected that most Boolean functions used in practice are vulnerable to this known $I V$ attack on a linear resynchronization mechanism.

\subsection{An Algebraic Attack}

As said in Sect. 5.2, the goal is to find a solution to the system of equations as described in (10). Again, the approaches to reduce the degree as described in Sect. 5.2 can be also applied here.

If all bits of $\mathrm{K}_{t}$ are uniquely specified by the equations, use Gröbner bases or the linearization method to solve (10) clock by clock. If the degree of the equations is low (e.g., Toyocrypt), it might be faster and require less $I V$ s than the approach described in Sect. 6.2.

\subsection{The Degree-1 Attack}

Another approach is to apply the methods described in Sect. 5.2 if $e=1$ is possible. In this case, we get at least one linear equation in the bits of $\mathrm{K}_{t}$ directly. If we repeat this for enough values of $t$ the corresponding $K$ can be reconstructed by solving a system of linear equations.

The exact effort depends on many parameters: the function $g$, the number $R$ of frames, the corresponding values of $\mathrm{IV}^{i}$ and $z_{t}^{i}$ and so on (see also Sect. 11). The number of operations is about $\left((\operatorname{dim} g)^{2} \cdot\left|\mathcal{M}_{d}(g)\right|+k\right) \cdot k+k^{3}$ or more general $\left(\beta(\varphi, d)^{3}+k\right) \cdot k+k^{3}$. This indicates that the approach might be feasible if the degree $d$ is small.

\section{$7 \quad$ Attacks on Combiners with Memory}

Many stream ciphers use their linear state in conjunction with a (small) nonlinear memory in order to avoid the trade-off between correlation immunity and nonlinearity for the combining function, see Rueppel [23].

In this section we demonstrate that resynchronization attacks can also be performed on stream ciphers with memory. Note that the known $I V$ attack of Sect. 6] can also be applied on combiners with memory. 


\subsection{A Standard Resynchronization Attack}

We will use the following model based on the general case of the combiners with memory:

$$
\begin{aligned}
S_{0}^{i} & =A \cdot K \oplus B \cdot I V^{i} \\
M_{0}^{i} & =\text { const } \\
S_{t+1}^{i} & =C \cdot S_{t}^{i} \\
M_{t+1}^{i} & =h\left(D \cdot S_{t}^{i}, M_{t}^{i}\right)=h\left(\mathrm{~K}_{t} \oplus \mathrm{IV}_{t}^{i}, M_{t}^{i}\right) \\
z_{t}^{i} & =f\left(D \cdot S_{t}^{i}, E \cdot M_{t}^{i}\right)=f\left(\mathrm{~K}_{t} \oplus \mathrm{IV}_{t}^{i}, E \cdot M_{t}^{i}\right) .
\end{aligned}
$$

In practice, some designs only start outputting key stream when $t=\mu$; this results in an improved diffusion of the key and the initialization vector into the nonlinear state. We will discuss both the cases $\mu=0$ and $\mu>0$. Note that in some designs $M_{0}^{i}$ is also dependent on the key and the $I V$. This can in our model be treated in the same way as the case $\mu>0$.

$\boldsymbol{\mu}=\mathbf{0}$. In the case $\mu=0$, the resynchronization attack can easily be adapted to work also with combiners with memory. We again describe the attack with $\varphi$ resyncs.

The first series of outputs can be written as $z_{0}^{i}=f\left(\mathrm{~K}_{0} \oplus \mathrm{IV}_{0}^{i}, M_{0}^{i}\right)$. Because $M_{0}^{i}$ is known, the attacker can recover $\mathrm{K}_{0}$ by exhaustive search. He can then determine $M_{1}^{i}$ for all the resyncs using (22). Now he knows all inputs to (23) for $t=1$ except $\mathrm{K}_{1}$, which he can again recover through exhaustive search, and so on. All complexities of this attack are exactly the same as for the case without memory. The only difference is that each step now consists of one evaluation of $f$ and of $\varphi$ evaluations of $h$.

$\boldsymbol{\mu}>\mathbf{0}$. The attacker does not know the initial contents of the $m$-bit memory $M$. Moreover, this memory is different for each resync. The attack now works exactly as above, except that the attacker will first have to guess the contents of $M$ at $t=\mu$. The time complexity of the attack now becomes $2^{\varphi \cdot m} \cdot\lceil n / \varphi\rceil \cdot 2^{\varphi}$ evaluations of the $f$ and $h$ function. As $\varphi$ and $m$ are quite small in most actual designs, the attacks are feasible.

Let's take as an example a combiner consisting of 5 LFSRs with total length 320 bits, and with 5 memory bits. The complexity of the resynchronization attack is then equal to $2^{36}$ function evaluations.

Practical Considerations of the Attack. As for the case without memory, we would like that the attack always works with $\varphi$ resyncs. At some times, we will have several possible values for $\mathrm{K}_{i}$. In a second phase, we cannot use the exhaustive search method of the memoryless case, because we would then have to try all possible values for updating the memory bits, which would increase the complexity enormously. 
This problem can be easily overcome by implementing the algorithm with a depth-first search. When at some time $t$ we have several possibilities for $\mathrm{K}_{t}$, we pick the first one and go to $t+1$. If we have no solution at time $t$, we go back to $t-1$ and try the next possibility there. When we have arrived at time $\lceil k / \varphi\rceil$, we have found a sufficient number of values and we check if we have found the correct key. Simulations indicate that when the number of resyncs $R$ is equal to or larger than $\varphi$, the attack will find the correct values very quickly and has to search very few states.

The same approach can also be used when the attacker disposes of less than $\varphi$ resyncs, i.e., $R<\varphi$. In the case $\mu>0$ this may even be advantageous from a complexity viewpoint, because we have to perform an exhaustive search over less than $\varphi$ initial memory states. But again the complexity of the search algorithm will increase exponentially with decreasing $R$, making this attack feasible only when $R$ is close to $\varphi$.

A particularity is the case when the Boolean output function $f$ is linear. In that case we don't get new information at each new resync, because all equations $z_{0}^{i}=f\left(\mathrm{~K}_{0} \oplus \mathrm{IV}_{0}^{i}, M_{0}^{i}\right)$ are equivalent. This problem can be easily overcome by using the memory update function $h$ to do the checks during the search. An example of such a linear output function is E0.

\subsection{Using Ad-Hoc Equations}

Another possibility is the use of ad-hoc equations which have been introduced in [3. The authors showed that for a combiner with memory with $m$ memory bits $M_{t}$, an equation

$$
F\left(S_{t}^{i}, \ldots, S_{t+m}^{i}, z_{t}^{i}, \ldots, z_{t+m}^{i}\right)=0
$$

of degree $\leq\left\lceil\frac{\varphi \cdot(m+1)}{2}\right\rceil$ always exists which is completely independent of the memory bits. They also propose an algorithm to find ad-hoc equations

$$
G\left(S_{t}^{i}, \ldots, S_{t+r-1}^{i}, z_{t}^{i}, \ldots, z_{t+r-1}^{i}\right)=0
$$

with the lowest possible degree $d$ if $\varphi \cdot r$ is not too large. For example, an ad-hoc equation of degree 4 using $r=4$ successive clocks exists for the $E_{0}$ key stream generator.

As (25) is independent of the memory bits, these equations can be used for all attacks described in the previous sections. An additional requirement is now that the attacker knows enough successive key stream bits.

If $\varphi \cdot r$ is small the Daemen et al. attack is applicable. The number of operations is about $\lceil k /(\varphi \cdot r)\rceil \cdot 2^{\varphi \cdot r}+k^{3}$. The methods described in Sect. 5.2 to reduce the degree of the equations can be easily adapted to the case of ad-hoc equations.

\section{Attacks on Stream Ciphers with Nonlinear Resynchronization}

In this section, we will show that stream ciphers with a nonlinear resynchronization mechanism can also be vulnerable to resynchronization attacks. A first 
attack is a chosen $I V$ attack; its principle is similar to that of Daemen et al. The second attack is a known $I V$ attack that uses the principle of linear approximations as used in the known $I V$ attack of Sect. 6.2. We will demonstrate these attacks on the two-level memoryless combiner. The framework of the attack is shown in Fig. 1, In a first level, the key and an $I V$ are linearly loaded into the LFSRs. The input to $f$ at time $t$ of the level 1 initialization is denoted $\mathrm{x}_{t}$. The following holds:

$$
\mathrm{X}_{t}=\left(x_{t}^{0}, x_{t}^{1}, \ldots, x_{t}^{\varphi-1}\right)=\mathrm{K}_{t} \oplus \mathrm{IV}_{t}=\left(k_{t}^{0} \oplus i v_{t}^{0}, k_{t}^{1} \oplus i v_{t}^{1}, \ldots, k_{t}^{\varphi-1} \oplus i v_{t}^{\varphi-1}\right) .
$$

The output $y_{i}$ of level 1 is collected and is used as the initial state for the level 2 generator. We use here the simplified setting shown in the figure. Level 2 generates the key stream $z_{i}$.

LEVEL 1

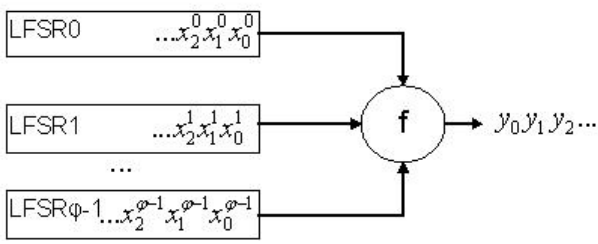

Fig. 1. Model for a two-level combiner

\section{LEVEL 2}

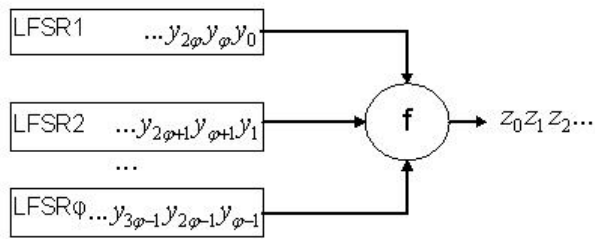

\subsection{A Chosen IV Attack}

We will show an attack scenario on this construction which holds under the assumption that the attacker can choose the value of the IVs which go into the Boolean function (this is for instance the case when the initial state equals the $\mathrm{XOR}$ of the key and the initialization vector).

We start with $t=0$. We let $\mathrm{IV}_{0}$ take $j$ different values, while keeping the other IVs constant. We obtain the following equations:

$$
\left\{\begin{array}{l}
f\left(f\left(\mathrm{~K}_{0} \oplus \mathrm{IV}_{0}^{1}\right), y_{1}, \ldots y_{\varphi-1}\right)=z_{0}^{1} \\
f\left(f\left(\mathrm{~K}_{0} \oplus \mathrm{IV}_{0}^{2}\right), y_{1}, \ldots y_{\varphi-1}\right)=z_{0}^{2} \\
\cdots \\
f\left(f\left(\mathrm{~K}_{0} \oplus \mathrm{IV}_{0}^{j}\right), y_{1}, \ldots y_{\varphi-1}\right)=z_{0}^{j} .
\end{array}\right.
$$

Denote the vector $\left(y_{1}, \ldots y_{\varphi-1}\right)$ by $\mathrm{R}$. In half of the cases, we will see that all $z_{0}^{i}$ are equal (either all 0 or all 1 ). This is due to the fact that $f(0, \mathrm{R})=f(1, \mathrm{R})$. In the other half of the cases, which is of interest here, the $z_{0}^{i}$ take both the values 0 and 1 in a random-looking way; which is due to the fact that $f(0, R) \neq f(1, \mathrm{R})$.

Assume that we are in the latter case. We now guess the $\varphi-1$ bits of R, let's call this guess G. If $f(0, \mathrm{G})=f(1, \mathrm{G})$, then our guess is certainly not correct and we proceed to the next guess. If $f(0, \mathrm{G}) \neq f(1, \mathrm{G})$, the guess is possible; we now get $j$ equations in $\mathrm{K}_{0}$ of the form:

$$
f\left(\mathrm{~K}_{0} \oplus \mathrm{IV}_{0}^{i}\right)=z_{0}^{i} \oplus f(0, \mathrm{G}) .
$$


The equations we have obtained are exactly the same as in the case of the linear resynchronization mechanism. If $j$ is large enough, we expect to find a unique solution for $\mathrm{K}_{0}$ over all the guesses for $\mathrm{G}$. It can be shown that we need about $2 \cdot \varphi$ chosen $I V$ s to achieve this.

In the same way, we can also recover $\mathrm{K}_{1}, \mathrm{~K}_{2}, \ldots$ which gives us the whole secret key of the system. The attack requires a total of about $2 \cdot k$ chosen $I V \mathrm{~s}$ and has a time complexity of $\lceil k / \varphi\rceil \cdot 2^{2 \cdot \varphi-1}$. This attack has been implemented on various 8-bit Boolean functions, and we can easily recover the key.

\subsection{A Known $I V$ Attack}

In this attack, we extend the approach in which we search a bias in the $\varphi$-input Boolean function (see the known $I V$ attack in Sect. 6) in a straightforward way to the case of the two-level combiners. Each bit now goes twice through the function $f$, but we can show that a bias still persists. The equation we want to hold is as follows:

$$
\bigoplus_{j \in B S} k_{j}=\bigoplus_{j \in B S} i v_{j}^{i} \oplus z_{0}^{i},
$$

where the set $B S$ consists of the bits of the set $S$ involved in the linear bias, for all times $t \in S$ of the first level of the combiner. Similar equations can be written for the next iterations. Let's denote the cardinality of the set $S$ by $s$. Of course it holds that $s \leq \varphi$. The cardinality of the set $B S$ is then evidently $s^{2}$.

The piling-up lemma [18] learns that the probability that this equation holds is equal to $1 / 2+2^{s} \cdot \epsilon^{s+1}$, where $\epsilon$ is the bias of the Boolean function. The bias of this equation is thus $2^{s} \cdot \epsilon^{s+1}$, which means we need $R=2^{-2 \cdot s} \cdot \epsilon^{-2 \cdot s-2}$ resyncs to break this system by a known $I V$ attack.

We will show what this implies for actual Boolean functions. We take Boolean functions with $\varphi$ inputs and resilience $\rho$. We will use two well-known lower bounds for the bias $\epsilon$ :

$$
\left\{\begin{array}{l}
\epsilon \geq 2^{-\varphi / 2-1} \\
\epsilon \geq 2^{\rho+1-\varphi}
\end{array}\right.
$$

where the first bound is due to Parseval's relation and the second to the trade-off between nonlinearity and resilience, see 28. The cardinality of the set $S$ is now 5 $s=\rho+1$. We can calculate an upper bound for the number of resyncs needed for a successful attack as a function of $\varphi$ and of the resilience $\rho$. This is shown in Fig. 2 for some values of $\varphi$. These graphs show that memoryless combiners with few inputs cannot be made resistant against resynchronization attacks on a twolevel combiner. For larger functions it should be checked whether the Boolean

\footnotetext{
${ }^{5}$ We conjecture that we will find the bias for $\rho+1$ in practice. We will certainly find a bias for $\rho+1$, as $f$ is $\rho$-th order correlation immune but not $\rho+1$-th order correlation immune. As the cases we discuss are optimal from a designer's point of view, we expect the Walsh spectrum to be flattened as much as possible over the values with Hamming weight $>\rho$ and therefore to find a bias (very close) to $\epsilon$ for Hamming weight $\rho+1$. This conjecture can be easily verified for a popular class of functions, the plateaued functions 31 .
} 
function is strong enough to withstand the above attack. The bounds given here may be refined by a more careful examination of the properties of the various classes of Boolean functions.

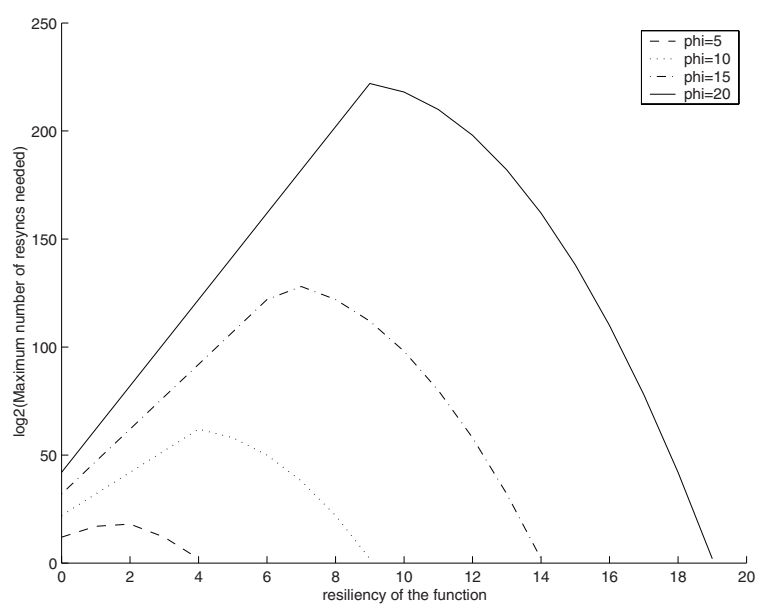

Fig. 2. Upper bound for the number of resyncs as a function of the resilience $\rho$ with as parameter the number $\varphi$ of input bits of the Boolean function

\subsection{Implications of the Attacks}

The known $I V$ attack described above for the two-level memoryless combiner can be easily extended to other nonlinear resynchronization mechanisms. It is also possible to apply the attack on other designs, such as combiners with memory, nonlinearly filtered generators and irregularly clocked shift registers. We can use techniques as described by Golic [13, 14 to find suitable linear approximations. Our attack can be used to evaluate the strength of any resynchronization mechanism, and resistance against this attack is a minimum requirement for any design. We are currently investigating the impact of this attack on some actual designs, such as the resynchronization mechanisms of E0 and the NESSIE [22] candidates.

\section{Conclusions}

In [10, Daemen, Govaerts and Vandewalle presented the original resynchronization attack on synchronous stream ciphers. In this paper, we have extended this resynchronization attack in several directions, by using new attack methods and by combining the attack with cryptanalytic techniques such as algebraic attacks and linear cryptanalysis.

Our attacks on linear resynchronization mechanisms show that a linear resynchronization mechanism should never be used in practice. Even if the system uses 
few resyncs, has an input function with many inputs and has a non-linear memory, it will still very likely contain weaknesses that can be exploited by one of our attack scenarios.

Nowadays, resynchronization mechanisms are typically designed in an ad hoc manner, by making them nonlinear to an extent that seems to be sufficient. Our attacks on nonlinear resynchronization mechanisms lead to a better understanding of the strength of such mechanisms and can be used to provide a lower bound for the nonlinearity required from a secure resynchronization mechanism. This allows designers to consider the trade-offs between the speed and the security of a resynchronization mechanism.

\section{Acknowledgements}

The authors would like to thank An Braeken, Joe Cho, Matthias Krause, Stefan Lucks, Erik Zenner and the anonymous referees for helpful comments and discussions.

\section{References}

1. R. Anderson, A5 (Was: Hacking Digital Phones), sci.crypt post, June 1994.

2. F. Armknecht, J. Lano, B. Preneel, Extending the Resynchronization Attack (extended version), Cryptology ePrint Archive, Report 2004/232, 2004.

3. F. Armknecht, M. Krause, Algebraic Attacks on Combiners with Memory, Crypto 2003, LNCS 2729, D. Boneh, Ed., Springer-Verlag, pp. 162-176, 2003.

4. F. Armknecht, Improving Fast Algebraic Attacks, FSE 2004, LNCS 3017, B. Roy, W. Meier, Eds., Springer-Verlag, pp. 65-82, 2004.

5. F. Armknecht, On the Existence of Low-degree Equations for Algebraic Attacks, Cryptology ePrint Archive, Report 2004/185, 2004.

6. Bluetooth S.I.G., Specification of the Bluetooth System, Version 1.2, available from www.bluetooth.org/spec, 2003.

7. Y. Borissov, S. Nikova, B. Preneel, J. Vandewalle, On a Resynchronization Weakness in a Class of Combiners with Memory, SCN 2002, LNCS 2576, S. Cimato, C. Galdi, G. Persiano, Eds., Springer-Verlag, pp. 164-173, 2002.

8. N. Courtois, W. Meier, Algebraic Attacks on Stream Ciphers with Linear Feedback, Eurocrypt 2003, LNCS 2656, E. Biham, Ed., Springer-Verlag, pp. 345-359, 2003.

9. N. Courtois, Fast Algebraic Attacks on Stream Ciphers with Linear Feedback, Crypto 2003, LNCS 2729, D. Boneh, Ed., Springer-Verlag, pp. 177-194, 2003.

10. J. Daemen, R. Govaerts, J. Vandewalle, Resynchronization Weaknesses in Synchronous Stream Ciphers, Eurocrypt 1993, LNCS 765, T. Helleseth, Ed., SpringerVerlag, pp. 159-167, 1993.

11. S. Fluhrer, Improved key recovery of level 1 of the Bluetooth Encryption System, Cryptology ePrint Archive, Report 2002/068, 2002.

12. C. Fontaine, Contribution à la Recherche de Fonctions Booléennes Hautement Non Linéaires, et au Marquage d'Images en Vue de la Protection des Droits d'Auteur, PhD Thesis, Paris University, 1998.

13. J. Golic, Correlation via Linear Sequential Circuit Approximation of Combiners with Memory, Eurocrypt 1992, LNCS 658, R. Rueppel, Ed., Springer-Verlag, pp. 113-123, 1992. 
14. J. Golic, Linear Cryptanalysis of Stream Ciphers, FSE 1994, LNCS 1008, B. Preneel, Ed., Springer-Verlag, pp. 154-169, 1994.

15. J. Golic, G. Morgari, On the Resynchronization Attack, FSE 2003, LNCS 2887, T. Johansson, Ed., Springer-Verlag, pp. 100-110, 2003.

16. A. Joux, F. Muller, A Chosen IV Attack against Turing, SAC 2003, LNCS 3006, M. Matsui, R. Zuccherato, Eds., Springer-Verlag, pp. 194-207, 2003.

17. D. Lee, J. Kim, J. Hong, J. Han, D. Moon, Algebraic Attacks on Summation Generators, FSE 2004, LNCS 3017, B. Roy, W. Meier, Eds., Springer-Verlag, pp. 34-48, 2004.

18. M. Matsui, Linear Cryptanalysis Method for DES Cipher, Eurocrypt 1993, LNCS 765, T. Helleseth, Ed., Springer-Verlag, pp. 386-397, 1993.

19. W. Meier, E. Pasalic, C. Carlet, Algebraic Attacks and Decomposition of Boolean Functions, Eurocrypt 2004, LNCS 3027, C. Cachin, J. Camenisch, Eds., SpringerVerlag, pp. 474-491, 2004.

20. W. Meier, O. Staffelbach, Correlation Properties of Combiners with Memory in Stream Ciphers (extended abstract), Eurocrypt 1990, LNCS 473, I. Damgard, Ed., Springer-Verlag, pp. 204-213, 1990.

21. M. Mihaljević, H. Imai, Cryptanalysis of Toyocrypt-HS1 stream cipher, IEICE Transactions on Fundamentals, vol. E85-A, pp. 66-73, Jan. 2002. Available at http://www.csl.sony.co.jp/ATL/papers/IEICEjan02.pdf.

22. New European Schemes for Signature, Integrity and Encryption, http://www.cryptonessie.org

23. R. Rueppel, Correlation Immunity and the Summation Generator, Crypto 1985, LNCS 218, H. Williams, Ed., Springer-Verlag, pp. 260-272, 1985.

24. R. Rueppel, Analysis and Design of Stream Ciphers, Springer-Verlag, Berlin, 1986.

25. M. Saarinen, Bluetooth und E0, sci.crypt post, February 2002.

26. P. Sarkar, S. Maitra, Nonlinearity Bounds and Constructions of Resilient Boolean Functions, Crypto 2000, LNCS 1880, M. Bellare, Ed., Springer-Verlag, pp. 515-532, 2000 .

27. V. Strassen, Gaussian Elimination is Not Optimal, Numerische Mathematik, vol 13, pp. 354-356, 1969.

28. Y. Tarannikov, On Resilient Boolean Functions with Maximum Possible Nonlinearity, Indocrypt 2000, LNCS 1977, B. Roy, E. Okamoto, Eds., Springer-Verlag, pp. $19-30,2000$.

29. G. Xiao, J. Massey, A Spectral Characterization of Correlation-immune Combining Functions, IEEE Trans. Inf. Theory, Vol. IT-34, pp. 569-571, 1988.

30. K. Zeng, C. Yang, T. Rao, On the Linear Consistency Test (LCT) in Cryptanalysis with Applications, Crypto 1989, LNCS 435, G. Brassard, Ed., Springer-Verlag, pp. 164-174, 1990.

31. Y. Zheng, X. Zhang, Plateaued Functions, ICICS 1999, LNCS 1726, V. Varadharajan, Y. Mu, Eds., Springer-Verlag, pp. 284-300, 1999. 